
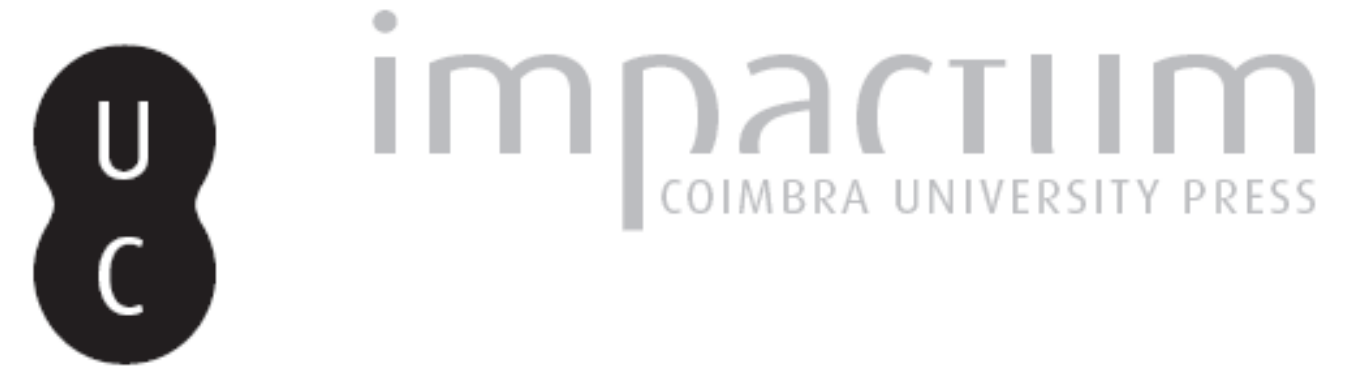

\title{
Ascendenze platoniche in Ensaio sobre a cegueira di José Saramago
}

Autor(es): $\quad$ Scotto, Giuliana

Publicado por: Imprensa da Universidade de Coimbra

URL persistente:

URI:http://hdl.handle.net/10316.2/42610

DOI:

DOl:https://doi.org/10.14195/0870-8584_3_7

Accessed : $\quad$ 26-Apr-2023 07:20:02

A navegação consulta e descarregamento dos títulos inseridos nas Bibliotecas Digitais UC Digitalis, UC Pombalina e UC Impactum, pressupõem a aceitação plena e sem reservas dos Termos e Condições de Uso destas Bibliotecas Digitais, disponíveis em https://digitalis.uc.pt/pt-pt/termos.

Conforme exposto nos referidos Termos e Condições de Uso, o descarregamento de títulos de acesso restrito requer uma licença válida de autorização devendo o utilizador aceder ao(s) documento(s) a partir de um endereço de IP da instituição detentora da supramencionada licença.

Ao utilizador é apenas permitido o descarregamento para uso pessoal, pelo que o emprego do(s) título(s) descarregado(s) para outro fim, designadamente comercial, carece de autorização do respetivo autor ou editor da obra.

Na medida em que todas as obras da UC Digitalis se encontram protegidas pelo Código do Direito de Autor e Direitos Conexos e demais legislação aplicável, toda a cópia, parcial ou total, deste documento, nos casos em que é legalmente admitida, deverá conter ou fazer-se acompanhar por este aviso.

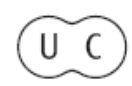




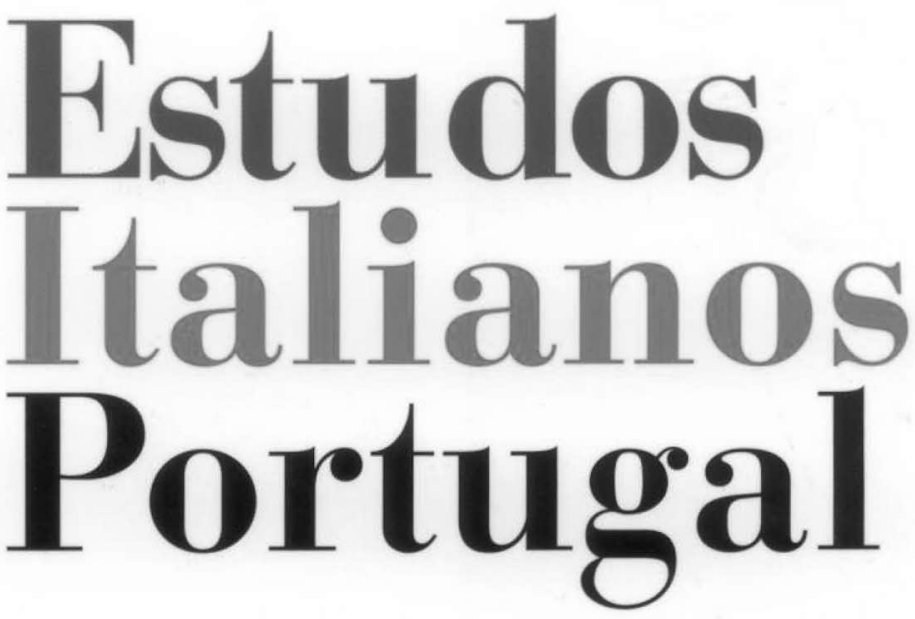

Instituto

Italiano

de Cultura

de Lisboa

Nova Série

$\mathrm{N}^{\circ} 3$

2008 


\title{
ASCENDENZE PLATONICHE IN ENSAIO SOBRE A CEGUEIRA DI JOSÉ SARAMAGO
}

\author{
Givliana Scotto*
}

Und die Lieb' auch heftet fleißig die Augen

F. Hölderlin, Andenken

SE SI PENSA a un rapporto possibile fra l'universo letterario di José Saramago e la filosofia di Platone, viene in mente quasi con banale ovvietà il romanzo A Caverna (2000) il cui titolo reca il riferimento al noto mito platonico.

Questo legame con la filosofia di Platone, in particolare con le idee esposte nella Repubblica, ma non soltanto, è invece meno evidente in Ensaio sobre a cegueira ma non per questo meno fondamentale. In questo breve scritto vorremmo mettere in luce, più di quanto non sia stato già fatto dalla letteratura critica ${ }^{1}$, la profonda ascendenza platonica che caratterizza anche questo romanzo dello scrittore portoghese.

* Laureata in Giurisprudenza e in Filosofia presso l'Università di Roma “Sapienza”. È dottore di Ricerca in Diritto Internazionale. È autrice di saggi in materie giuridiche e letterarie, fra cui, con Antonio Marchesi, Il diritto dell'Unione europea (2005). Attualmente è docente presso la Facoltà di Filosofia dell'Università "Sapienza" nonché assegnista di ricerca in traduzione giuridica presso la Facoltà di Lettere e Filosofia della stessa Università. Su José Saramago ha pubblicato due articoli, "Saramagos Stadt der Blinden und Platons Staat" (2005), e "Saramagos As intermitências da morte, Ein Märchen für Erwachsene" (2007), comparsi in Matices. Zeitschrift zu Lateinamerika, Spanien und Portugal.

1 Un cenno di questo legame con la filosofia platonica si coglie per es. in T. C. Cerdeira da Silva, "De cegos e visionários: uma alegoria finissecular na obra de José Saramago", in Cânones E Contextos, Congresso Abralic, Anais, v. 3, Rio de Janeiro, UFRJ, 1998, pp. 691-694, alle pp. 692-693; R. Ammicht Quinn, "Versuch über die Blindheit José Saramagos Stadt der Blinden und die Situierung der Literaturethik im gesamtethischen Diskurs", in A. Holderegger, 


\section{Politica}

Accostando L'Ensaio alla Repubblica un primo aspetto comune che balza agli occhi è la tematica politica.

In vari punti del romanzo di Saramago la questione politica emerge con evidenza. Sin dalla fase iniziale della vicenda l'azzeramento del grado del vivere civile provocato dall'epidemia di cecità rende necessario un riassetto dei rapporti all'interno della nuova comunità di ciechi. Non appena il primo gruppo di ciechi si installa nella camerata dell'ex-manicomio, il medico viene nominato "responsabile" 2 , analogamente a quanto accadrebbe all'origine della costituzione di un gruppo sociale allorché viene designato un "capo". Anche la combriccola di ciechi organizzatisi per sopraffare gli altri ricoverati costituisce una cellula di aggregazione politica ${ }^{3}$, con la previsione al suo interno di un principio di divisione delle funzioni al fine di mantenere l'ordine ${ }^{4}$. Ancora, l'incendio dell'ex-manicomio che consente la fuga dei protagonisti assume significato politico in quanto esemplifica una regola storica: la celere destabilizzazione di un sistema politico palesemente ingiusto come quello istituito dal gruppo di ciechi malvagi ${ }^{5}$.

J.P. Wils, Interdisziplinäre Ethik. Grundlagen, Methoden, Bereiche. Festschrift Dietmar Mieth, Freiburg i.Br. 2001, pp. 269-287; D. Frier, "Righting Wrongs, Re-Writing Meaning and Reclaiming the City in Saramago's Blindness and All the Names", in PLCS, 6, 2001, pp. 97-122, a p. 97.

${ }^{2}$ J. Saramago, Ensaio sobre a cegueira (1995) Lisboa, Caminho, 9. ${ }^{a}$ ed. (d'ora in poi: Ensaio), p. 53.

3 Ensaio, pp. 137 ss.

${ }^{4}$ Funzione di gestione del cibo, sorveglianza, contabilità: v. Ensaio, pp. 14 e 159.

5 Ensaio, pp. 205 ss. In termini assai simili la lettura che lo stesso Platone offre del fenomeno della città ingiusta in Rep., 351d-352a. Un'osservazione vicina alla direzione qui accolta ma che, a nostro avviso, risuona troppo semplicistica si coglie in V. Maurya, "Construction of Crowd in Saramago's Texts", in Colóquio/Letras, vol. cit., pp. 267-278, a p. 267, che considera la vicenda come lotta di un gruppo contro un assetto sociale ingiusto. 
Una coloritura politica è anche quella che si distende sulla convivenza dei personaggi insediatisi nella casa del medico e di sua moglie una volta fuggiti dall'ex-manicomio ${ }^{6}$. La convivenza nell'appartamento del medico ha $\mathrm{i}$ tratti della comune, i rapporti sono impostati su base paritaria ${ }^{7}$, pur nel limite della situazione di cecità (o di vista, nel caso della moglie del medico) che li sovrasta.

Come nella Repubblica di Platone, vagliati vari sistemi politici ingiusti ${ }^{8}$ si giunge alla riflessione sulla città giusta per natura ${ }^{9}$, nella città colpita dalla cecità, una volta passati per varie tipologie di aggregazione politica, transeunti proprio a partire dal fondamento della cecità ${ }^{10}$, si giunge all'instaurarsi della comunità dei sette protagonisti del romanzo, dei quali la moglie del medico, unica vedente, è naturale e necessaria guida.

A questo punto si innesta un'altra analogia fra il romanzo-saggio di Saramago e la riflessione del filosofo.

\section{Vedere}

Il fondamento in cui la tematica politica finisce col radicarsi e che più profondamente accomuna $i$ due testi è infatti il vedere $^{11}$.

6 Ensaio, pp. 245 ss.

7 La moglie del medico trova in casa "o necessário para cobrir com decência sete pessoas, é verdade que não eram todas da mesma estatura, mas na magreza pareciam gémeas" (Ensaio, p. 261). Un analogo rapporto di fratellanza informa la città platonica: Rep., 415a.

8 Rep., Libro VIII, 546 ss.

9 Rep., 582d ss.

10 I. Pires de Lima, "Dos 'anjos da história' em dois romances de Saramago" in Colóquio/Letras, cit., pp. 415-26, a p. 418, afferma che le persone colpite dalla cecità sono "incapazes de qualquer forma de organização social".

11 In particolare la Ammicht Quinn nel suo saggio su Ensaio sobre a cegueira parla di "metafora del vedere" (passim). 
Nel romanzo di Saramago il tema del vedere viene in primo piano perché la protagonista - la moglie del medico continua a vedere per tutto il romanzo; viene in rilievo per l'aspetto negativo, perché i ciechi sono costretti in una privazione della vista che viene a mancare loro come se mancasse il fondamento della vita di relazione abituale ${ }^{12}$.

Una relazione analoga fra vedere e non-vedere fonda, nella Repubblica platonica, il rapporto fra il filosofo, che ha visto il vero/il bene/il bello, e chi invece continua a muoversi fra le ombre che popolano la caverna di cui Platone parla spiegando la condizione del filosofo in termini mitici ${ }^{13}$.

Nella Repubblica platonica così come nell'Ensaio il rapporto fra il vedere e il non-vedere si radica in una divisione che taglia in due la comunità politica di cui si parla in ciascuno dei due testi. Nella Repubblica la divisione separa i filosofi dalle altre classi sociali formanti la città ${ }^{14}$. Nell'Ensaio la divisione isola dalla gran massa dei ciechi l'unica donna che mantiene la vista e che per questo è posta a capo della piccola comunità di fuggiaschi.

Quasi a incarnare la riflessione platonica, nella figura della moglie del medico confluiscono le aspettative che una società ripone nei suoi intellettuali, quelli che per maggior capacità di riflessione o senso di responsabilità dovrebbero sapere dove stiamo andando, dovrebbero comprendere e aver cura degli altri intorno a sé. Ma come la città platonica si fonda sull'essere di ciascuno, concepito come limite invalicabile, anche i personaggi di Saramago sono privati del loro nonessere, della possibilità di esser-altro, e sono posti a contatto

12 Dei cinque sensi la vista è quello più sviluppato e il conoscere teoretico, su cui si basa tutta la nostra cultura occidentale sin dagli antichi greci, è un guardare. Sul punto v. M. Heidegger, Vorträge und Aufsätze, Neske, Pfullingen, 1954, tr. it. Saggi e discorsi, Mursia, Milano, 1976, pp. 31-36.

13 Rep., 515c ss.

14 Rep., 476a-b. 
con il proprio limite. Per es. la moglie del medico dichiara l'integrità della propria vista come una pena, perché da ciò che vede non può trovar rifugio, lei vede e questo è il suo limite, non ha protezione nel mare di latte della cecità che ha colpito gli altri, i quali non possono vedere e questo è il loro limite. Tanto per Platone che per Saramago questo limite fondato sull'essere di ciascuno si opacizza ricongiungendosi al non-essere che ci costituisce come esistenti non appena ci si riaccosti alla realtà concreta: Platone è consapevole che la città retta dai filosofi non esiste ${ }^{15}$; l'Ensaio si chiude con l'unificarsi di vedere e non-vedere nello sguardo della moglie del medico davanti alla finestra.

\section{Amore}

Alla questione del vedere Platone intreccia nella sua Repubblica un'altra tematica, peraltro in questo testo soltanto accennata $^{16}$, mentre è oggetto di un altro grande dialogo, il Simposio. Il filosofo non soltanto è colui che ha visto, ma è anche colui il cui agire è mosso dall'amore ${ }^{17}$. Nella riflessione platonica l'amore filosofico rappresenta il grado supremo di amore. Dunque il filosofo è colui che in sommo grado vede e ama.

Oltre la vicinanza che può ravvisarsi fra la città platonica e la città dei ciechi per quanto riguarda il tema politico, la concezione dell'amore che sorregge la riflessione platonica permette di enucleare quello che a nostro avviso è il tema fondamentale del romanzo di Saramago.

Questo avvincente romanzo che narra le vicende di ciechi, del loro spaesamento, che parla di crudeltà, di sporcizia, di violenza, in realtà, sotto la dura scorza della drammaticità

15 Rep., 592a-b.

16 Rep., 474c-475e.

17 Platone, Simposio, 204a-b. 
della vicenda, parla dell'amore. L'amore è, in questa forma dissimulata, il tema più importante. Ensaio sobre a cegueira dice che l'amore è raro, ma quando c'è, quando è veramente amore, è compassione smisurata, è attenzione, benevolenza, gentilezza, pazienza, comprensione, assistenza, sacrificio, è compromettersi nel nome di una fiducia nell'essere umano in quanto tale. Una dimensione di universalità - una sorta di Menschlichkeit - costituisce l'aspetto fondamentale dell'amore su cui batte l'accento Saramago, perché soltanto se incontriamo l'altro in quanto essere umano la dignità dell'altro viene garantita e protetta. Un amore senza rispetto ${ }^{18}$ non è un amore abbastanza profondo, è un amore cieco, perché non vede l'altra persona in quanto tale.

Il tema del vedere si intreccia dunque a quello dell'amore. La moglie del medico, che ha compassione per chiunque, è colei che vede e continua a vedere. Il modo stesso in cui invece gli altri personaggi perdono la vista sembra andare di pari passo con la loro grettezza, con la loro diffidenza e sfiducia.

L'amore va assieme alla vista e sono ciechi coloro che sono privi d'amore, in quanto privi d'amore. Non che i ciechi non abbiano sentimenti, non che non sappiano essere "buoni": ci affezioniamo a tutti i personaggi del romanzo, su nessuno osiamo levare un giudizio di condanna inappellabile, ma i ciechi sono privi dell'amore rispettoso, della gentilezza e della carità compassionevole che invece animano la moglie del medico. C'è come un salto fra l'amore, nella incondizionatezza che caratterizza il modo della moglie del medico, e i gesti anche buoni e generosi dei ciechi. C'è un salto e la differenza fra l'uno e l'altro modo è tracciata dal dono della vista o dalla sua perdita.

Il tema dell'amore intride così profondamente il romanzo da non potersi però esaurire nel suo aspetto universalistico.

18 Dal lat. spicio, guardo. 
Benché vi sia un salto fra il personaggio della donna che vede e gli altri, ciechi; e benché questo salto sia dato dalla qualità dell'amore che la donna profonde in ciò che fa e in ciò che pensa, è l'amore in tutte le sue sfaccettature, anche nelle sue manifestazioni più piccole e fragili, a pervadere il romanzo, dall'inizio alla fine. Non soltanto perché alcuni personaggi lungo la storia riescono a fondare un amore costruttivo, ma piuttosto perché nelle varie situazioni le rappresentazioni dell'amore, come affetto e tenerezza, così come quelle dedicate alla sessualità (fra cui l'orgia violenta), sono come rivoli e propaggini dell'amore, sue inquietanti manifestazioni che, aggrappandoci al punto di vista lucido della moglie del medico diremmo "amore in tono minore" o "storture", ma che in verità fanno parte della nostra esperienza più comune di accesso all'amore.

Per quanto appena detto non si può però concludere che in questa concezione l'amore sia connotato in senso puramente caritatevole. E ciò per almeno due motivi.

Il primo è che il delitto che, fra i tanti che costellano il romanzo, è per certi versi quello più inescusabile perché commesso in piena lucidità, viene perpetrato proprio dalla donna che vede, quando invece da lei non ci aspetteremmo mai la presa in carico di un gesto che dà la morte. Colui che viene ucciso dalla donna, con un'atroce forbiciata nella gola, nel momento culminante del godimento sessuale durante la violenza collettiva, è il capo del gruppo di ciechi che vuole organizzare in forma dispotica la convivenza dei ricoverati all'interno dell'ex-manicomio. Le circostanze portano forse a scusare questo omicidio quale atto di difesa se non di giustizia, ma questa resta una giustizia del tutto contingente e umana. Il gesto assassino, compiuto dalla mano più amorosa, non soddisfa, lascia nell'inquietudine. Sebbene in tal modo venga suggellata l'emancipazione da un potere ingiusto e crudele che si è imposto in modo antidemocratico, non si può fare a meno di pensare che que- 
sto tragico epilogo appartiene a una mera contingenza, perché anche quest'uomo che viene ucciso, per quanto forse più forte e più intelligente di altri internati, non è che un cieco. Ha una sorta di scusante che la donna che vede e che lo uccide non ha, perché su di lei grava la responsabilità della coscienza. Questo delitto getta un'ombra quanto mai sinistra sulla figura della donna che ha dalla sua l'amore, e per via di questo delitto inquietante, consumato al culmine dell'amore fisico fatto con violenza, il romanzo non può incanalarsi nella semplicistica direzione di un buonismo premiato, di una netta scissione fra ciechi "cattivi" e donna che vede "buona"19. La donna in cui si raccoglie l'amore è al contempo colei che compie il delitto per il quale forse, fra tutte le crudeltà e gli egoismi raccontati nella vicenda, meno si può dare giustificazione. Mentre gli altri delitti e gesti malvagi trovano la comprensione dello sguardo compassionevole della moglie del medico, il delitto di questa non trova nessuno sguardo, nessun giudice clemente. Resta senza sentenza, è un atto di pura responsabilità affidato al vuoto, consegnato alla mancanza di ragione da cui è scaturito il dramma della cecità.

Ma v'è un altro motivo per cui ci parrebbe di fraintendere Saramago se si riducesse l'amore a buona lezione di carità, magari di carità cristiana - la prima forma di amore incondizionato a cui pensiamo è in effetti l'insegnamento del Vangelo, e l'amore che testimonia la moglie del medico, dell'amore evangelico effettivamente presenta alcuni tratti. Per via di questo aspetto si potrebbe trascinare l'interpretazione del romanzo verso la sfera reli-

19 A proposito del tratto inquietante che in Saramago caratterizza la bontà, v. Alípio Maia e Castro, "No Diário de Saramago: um Humanismo Latente, Introdução", in Revista Internacional d'Humanitats, 1, 1998, disponibile in rete al sito http://www.hottopos.com.br/rih1/saramago.htm, anche in inglese, con il titolo “The Labyrinth of José Saramago's Religious Speculation”, reperibile in: http://www.hottopos.com.br/rih1/saramaen.htm. 
$\operatorname{giosa}^{20}$. Ma la scena dell'ingresso nella chiesa dove le statue sono bendate toglie questo conforto ${ }^{21}$. Benché si inserisca nella narrazione come deus ex machina, come cesura, a ricucire la spaccatura aperta dal dire proprio dell'opera, la descrizione delle statue sacre bendate nella chiesa a spiegazione ${ }^{22}$ dell'epidemia di cecità bianca colpisce come una denuncia rivolta nei confronti del divino, come atto di accusa contro Dio e il suo corteo di santi che chiudono gli occhi davanti alle umane debolezze e agli umani bisogni; ovvero, se gli occhi sono stati coperti da qualcuno o da qualcosa di esterno (in effetti non si dice come mai le statue siano bendate, né si capisce chi le abbia private della vista), resta l'amaro della riflessione rivolta al divino come un che di limitato ${ }^{23}$, che non è in grado di togliersi le bende dagli occhi e prendersi cura delle proprie creature.

L'amore in Ensaio è, pur nella sua grandezza, un amore tutto umano ${ }^{24}$.

In Ensaio l'amore assume mille forme e sfaccettature, ma pur nello sforzo sovrumano di comprensione e di apertura

${ }^{20}$ Per alcune riflessioni su temi di Ensaio sobre a cegueira comuni alla religione cristiana dal punto di vista di quest'ultima v. L. da Silva Pereira, "O Nobel de Saramago”, in Brotéria, 3, 148, Março 1999, pp. 263-274, alle pp. 270-271.

21 Ensaio, pp. 299 ss.

22 Si tratta in verità di una spiegazione per analogia, come di una rappresentazione speculare della rete dei rapporti posti fra le statue dagli occhi bendati e gli unici occhi vedenti che vengono offerti su un piatto, e che riproduce in qualche modo l'offerta che la moglie del medico fa della propria vista a vantaggio di coloro che non vedono.

23 A tale propostito v. Alípio Maia e Castro, "No Diário de Saramago", e i commenti relativi ai passi rilevanti dei Cadernos de Lanzarote di Saramago.

${ }^{24}$ Significativo il dialogo fra la moglie del primo cieco e la moglie del medico mentre si lavano nude sul terrazzo sotto la pioggia battente: "Só Deus nos vê, disse a mulher do primeiro cego, que, apesar dos desenganos e das contrariedades, mantém firme a crença de que Deus não é cego, ao que a mulher do médico respondeu, Nem mesmo ele, o céu está tapado, só eu posso ver-vos" (Ensaio, pp. 266-267). 
e di accoglienza compiuto da parte della donna che vede, si profila in qualche modo come amore possibile, come amore umano da cui il sostegno divino resta tagliato fuori. Una strada che, per quanto impervia, per quanto aspra possa mostrarsi, viene ciononostante additata - anche grazie ai frequenti commenti dell'io narrante - come strada praticabile. 